\title{
e-Phaïstos
}

e-Phaïstos Revue d'histoire des techniques / Journal of the history of technology

IX-2 | 2021

Quel objet pour quel musée?

\section{Le musée comme compendium du monde}

L'objet en représentation, le visiteur en liberté

The Museum as a Collection of the World: Representation for the Object, Freedom for the Visitor

Bruno Jacomy

\section{(2) OpenEdition}

\section{Journals}

Édition électronique

URL : https://journals.openedition.org/ephaistos/9295

DOI : 10.4000/ephaistos.9295

ISSN : 2552-0741

Éditeur

IHMC - Institut d'histoire moderne et contemporaine (UMR 8066)

Référence électronique

Bruno Jacomy, «Le musée comme compendium du monde », e-Phaïstos [En ligne], IX-2 | 2021, mis en ligne le 26 octobre 2021, consulté le 28 octobre 2021. URL : http://journals.openedition.org/ephaistos/ 9295 ; DOI : https://doi.org/10.4000/ephaistos.9295

Ce document a été généré automatiquement le 28 octobre 2021.

Tous droits réservés 


\section{Le musée comme compendium du monde}

L'objet en représentation, le visiteur en liberté

The Museum as a Collection of the World: Representation for the Object, Freedom for the Visitor

Bruno Jacomy

1 Dans la classe de l'école élémentaire de nos grands-parents trônait, au mur, une petite armoire vitrée en bois dans laquelle était présenté un ensemble d'instruments de mesure, témoins de l'uniformisation des unités sur tout le territoire national, un héritage important de la Révolution française. Elle comprenait en général : un mètre pliant, une chaîne d'arpenteur, un double-décimètre, un décimètre cube démontable, des mesures à vin, à lait, à huile, des mesures en bois, une balance Roberval, des poids en fonte et en cuivre. Ce compendium métrique, car tel était son nom savant, adressait aux écoliers un message scientifique doublé d'un manifeste civique et économique: celui de l'unité politique de la France, l'universalité de la science, la force de l'éducation de tous les petits Français... Cette mission universelle était concentrée en un lieu unique, avec des objets à regarder, observer, manipuler, et qui relayaient le message transmis par le maître ou la maîtresse d'école. 
Fig.1. Compendium métrique de l'école élémentaire reconstitué

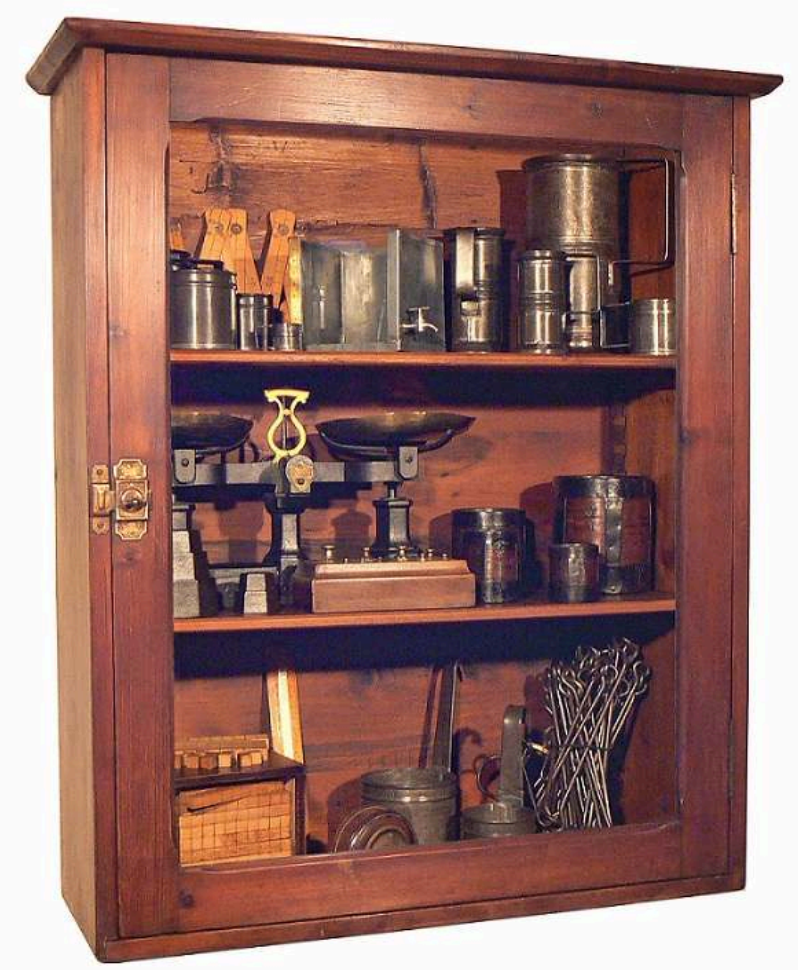

Cette petite armoire vitrée, destinée à l'apprentissage du système métrique décimal, est présente dans un grand nombre d'écoles élémentaires tout au long du XIXe siècle et pendant une bonne partie du $X X^{e}$.

Le Compendium/Albert Balasse/lecompendium.fr

2 Ce compendium est en quelque sorte le symbole du musée par son unicité et son universalité. Lui aussi expose des objets dans des vitrines dans le but de transmettre de l'information, mais surtout des savoirs, des savoir-faire, une ou des cultures, sans oublier du plaisir. Certes la réception de tout ceci par l'individu qui regarde la vitrine peut être très variée et susciter intérêt, curiosité, désir, ou bien encore ennui, incompréhension, doute. J'invite le lecteur de cet article, comme je le ferais avec un visiteur dans une exposition, à déambuler dans ce monde en miniature qu'est le musée à travers un cheminement en quatre étapes.

3 À l'image de la petite vitrine de la salle de classe, le musée est une représentation, un spectacle, où ni les objets ni les discours ne sont neutres, même s'ils visent à rester objectifs. Cette représentation n'est pas la réalité, elle en est une reconstruction, la mise en scène d'objets bien réels. Dès lors se pose la question de l'authenticité. Cette machine a-t-elle fonctionné, ce fronton de plâtre est-il une copie ou un objet historique, cette maquette pédagogique est-elle une œuvre ou un artifice muséographique...? Aux deux bouts de la chaîne, chacun -le concepteur d'un côté, le spectateur de l'autre-- joue le rôle d'interprète. Entre les deux se situe l'objet exposé, que ce soit une œuvre, un modèle, un spécimen ou une manip.

4 Dans le musée, la principale contrainte pratique est celle de l'espace. Qu'il s'agisse d'un bâtiment existant, avec ses volumes, ses conditions d'éclairage, ses circulations, ou d'un bâtiment neuf, telle une page blanche à habiter, l'organisation de cet espace fait partie de l'histoire que le visiteur retiendra à l'issue de son passage. Le lieu de l'action, de la 
représentation, est une enceinte bien délimitée dans laquelle une personne conservateur, commissaire d'exposition- a disposé des objets selon un ordre, une organisation reflétant un parti pris, conscient ou pas. Au-delà du concepteur, le scénographe ou le muséographe, l'éclairagiste et le socleur contribuent tous, à leur niveau, à donner au lieu, aux vitrines, à la présentation des objets, une ambiance, un "style", comme le dit l'ethnologue et muséographe Jacques Hainard ${ }^{1}$. La manière même dont les publics s'approprieront la visite découlera en grande partie des choix faits dans la mise en scène. Toutefois, même si le concepteur d'une exposition, le créateur d'un musée peut proposer un sens à la visite -sens chronologique, antichronologique, thématique- le visiteur, au bout du compte, sera seul maître du parcours qu'il va effectuer, de l'histoire qu'il va construire pendant le temps de sa visite. Lieu public, le musée est un lieu de liberté, ouvert à tous, où chacun peut prendre ou apprendre ce qu'il veut, dans l'ordre qui lui convient le mieux, dans le temps qu'il décide de consacrer à sa visite. Mais, paradoxalement, ce lieu public est aussi le lieu d'une intimité entre un objet et un individu, l'occasion d'une « conversation », comme le suggère le muséologue Jorge Wagensberg².

\section{L'univers en représentation}

5 La question de la représentation a déjà été clairement exprimée notamment par le sociologue Jean Davallon, il y a plus de trente ans, lorsqu'il présentait l'exposition comme « un modèle du monde réel », qui « opère une réduction et une modélisation du monde » (Davallon 1986 : 271). Mais si les gens de musée s'accordent généralement sur le fait qu' " une exposition doit être à la fois une thèse et un spectacle ${ }^{3}$ ", les visiteurs n'en sont pas forcément conscients. Institution de référence, le musée est perçu comme dépositaire d'un discours objectif, s'appuyant sur des collections dont l'authenticité n'est pas à mettre en doute. Or, ces notions n'ont rien d'absolu et dans ce spectacle qu'est l'espace d'exposition, il y a, comme au théâtre ou au cinéma, une multitude d'intervenants; du producteur au "consommateur», du concepteur au visiteur, en passant par les restaurateurs, taxidermistes, éclairagistes, scénographes, etc.

Je m'appuierai ici sur deux expériences vécues au musée des Confluences à Lyon: l'exposition Dans la Chambre des merveilles, l'une des expositions inaugurales, et la salle Sociétés du parcours permanent. Dans ces deux cas, la représentation était un élément clé du projet, sujet dans le premier, mode d'appréhension de l'espace dans l'autre. 
Fig.2. Exposition Dans la chambre des merveilles, présentée au musée des Confluences, à Lyon, du 20 décembre 2014 au 31 juillet 2016

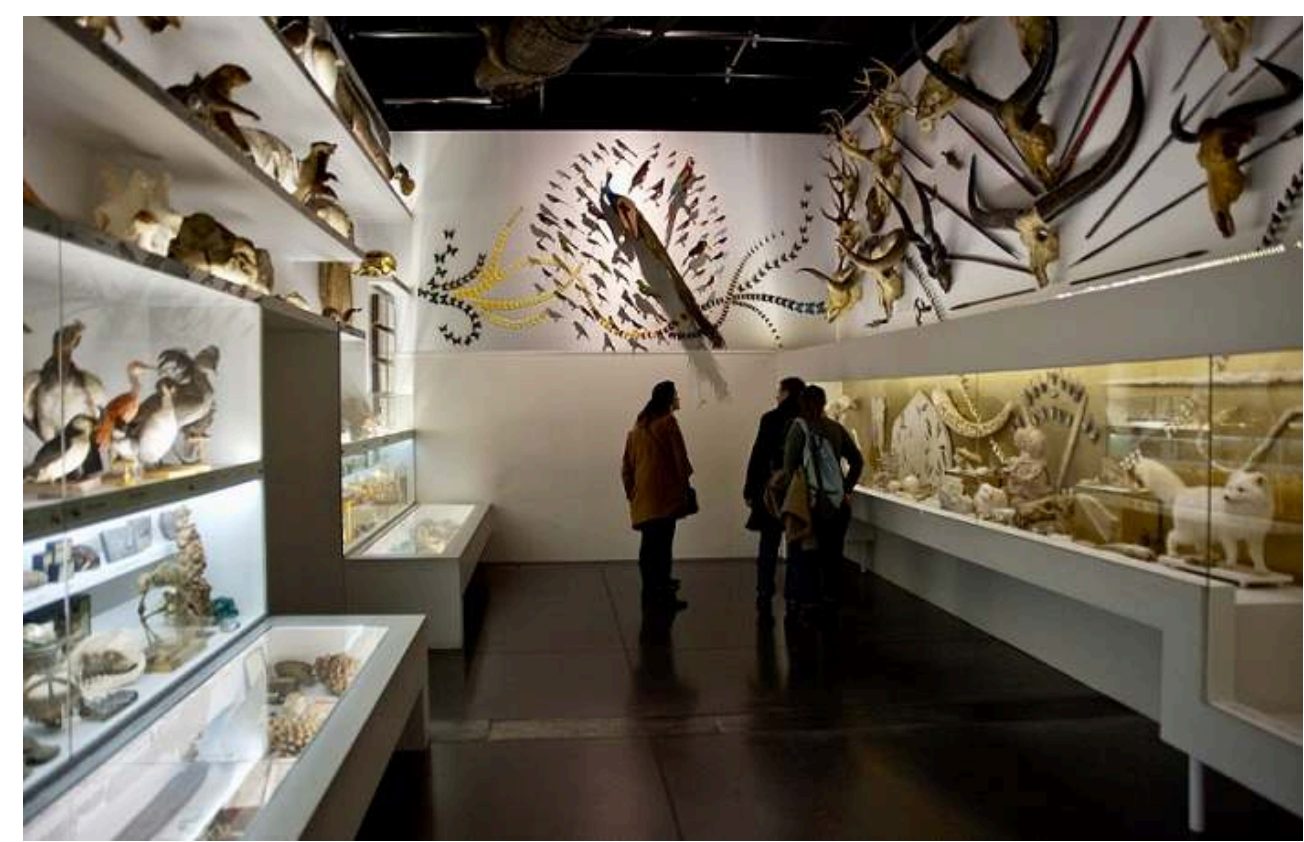

Cette réinterprétation d'un cabinet de curiosités du XVIIe siècle visait à ancrer le nouveau musée des Confluences dans son histoire et à le définir comme « un lieu où les frontières entre les savoirs s'effacent, un lieu où l'on vient certes pour apprendre mais aussi pour s'interroger et s'émerveiller » (dossier de presse de l'ouverture, décembre 2014).

Musée des Confluences, Lyon

7 L'exposition Dans la Chambre des merveilles ${ }^{4}$ avait pour objectif de raconter l'histoire même de l'institution, depuis les premiers cabinets du XVII ${ }^{e}$ siècle jusqu'à la mutation du muséum d'histoire naturelle vers le musée des Confluences, en passant par un musée colonial et le musée Guimet. Le fil conducteur de l'exposition était le personnage de Balthasar de Monconys (1611-1665), un magistrat ayant parcouru l'Europe à partir de 1645 pour constituer à Lyon, avec son frère Gaspard, un cabinet de curiosités qui fut alors fort prisé. La commande de départ de l'exposition était aussi de donner une idée de ce que pouvait être un cabinet de curiosités. Le matériau ne manquait pas, la collection du musée des Confluences en matière de sciences naturelles étant extrêmement riche, mais la difficulté consistait surtout à restituer l'atmosphère du cabinet d'un " curieux » du XVII siècle, sachant que la collection de Monconys avait été dispersée, que l'architecture intérieure d'aujourd'hui n'a rien à voir avec celle d'un hôtel particulier du XVII siècle et que les conditions d'exposition des collections, du point de vue des contraintes de climat et de sécurité notamment, empêchaient une présentation du même type que celle d'il y a plus de trois siècles. L'hypothèse d'une reconstitution fidèle d'un tel cabinet a été écartée aussi en raison du regard même que des visiteurs du XXI ${ }^{\mathrm{e}}$ siècle pouvaient porter sur une réalité depuis longtemps disparue. Si la littérature parle directement à l'imaginaire, le musée et l'exposition, comme d'ailleurs le théâtre ou le cinéma, donnent à voir une recomposition, métamorphosée par ces filtres de la représentation. Le parti a dès lors été pris, d'une part de travailler étroitement avec la scénographe sur une mise en scène traduisant les notions de profusion et d'émerveillement d'un cabinet, d'autre part d'arriver à ce cabinet à l'issue d'un parcours initiatique et pédagogique ménageant l'effet de surprise final. 
8 Les différentes étapes de ce parcours voulaient montrer aux visiteurs comment on était passé alors de l'accumulation à l'organisation rationnelle, de la curiosité à la science émergente ; il s'agissait aussi de mettre le visiteur dans la situation, devant des objets étranges, d'imaginer ce qu'un esprit curieux du milieu du XVII ${ }^{e}$ siècle pouvait bien ressentir et quelles théories il pouvait échafauder. Dans ce cheminement, il fallait enfin évoquer les voyages eux-mêmes ainsi que les indispensables échanges épistolaires et physiques des savants et curieux de l'époque.

9 L'exposition a eu un succès considérable, tant par sa fréquentation que par la satisfaction des publics, en grande partie parce que la découverte de cette salle, recouverte du sol au plafond de collections d'histoire naturelle, était en soi une œuvre plastique spectaculaire et replongeait le visiteur dans les traces que l'ancien muséum avait laissées dans les mémoires des parents et grands-parents. Mais ce succès est aussi dû au fait que l'exposition s'est construite avec la complicité des scénographes, intervenus assez tôt dans le projet pour participer au choix des objets et à leur disposition.

10 Au bout du compte, l'exposition n'était ni neutre, ni fidèle, ni même authentique. En revanche, les objets l'étaient et l'état d'esprit de nos prédécesseurs se voulait restituer avec sincérité. Dans cette exposition, l'objet était bien de montrer combien les cabinets de curiosités étaient en soi des abrégés du monde ${ }^{5}$, des lieux clos censés rassembler la diversité des richesses de notre planète à travers le regard des curieux du XVII ${ }^{e}$ siècle.

11 À l'inverse de l'exemple précédent, dans lequel l'objet était le concept de monde en réduction, le chapitre du parcours permanent intitulé Sociétés ${ }^{6} s^{\prime}$ appuie sur ce concept dans la mise en espace de l'exposition, dont l'ambition est de sensibiliser les visiteurs à la diversité des cultures humaines. Ce chapitre a pour sous-titre Le théâtre des Hommes et, dès que le visiteur pénètre dans la salle, il est en mesure d'embrasser du regard la totalité de la présentation. Pas de parcours proposé ni imposé, mais trois titres en gros caractères scandent le propos : organiser, échanger, créer. Cette partie de l'exposition permanente est sans conteste celle qui suscite le plus de questions et de réactions radicales : elle est honnie ou adorée. On y voit, en entrant, d'un côté un ensemble d'appareils ménagers récents, de l'autre des toiles aborigènes d'Australie, devant nous des boucliers africains, derrière eux un accélérateur de particules et un moteur de camion, mais aussi un métier à tisser, une armure de samouraï, une robe de mariée en fibre optique et une collection de minéralogie. Ce qui peut n'apparaître au premier abord que comme un gigantesque bric-à-brac est en fait le résultat de plusieurs années de réflexion, d'échanges, d'essais et d'erreurs, pour arriver à transmettre un discours sur la structuration des sociétés humaines, "entre rêves et utopies, révolutions et inventions » (musée des Confluences, Guide : 155). 
Fig.3. Vue de la salle Sociétés, le théâtre des Hommes, dans le parcours permanent du musée des Confluences

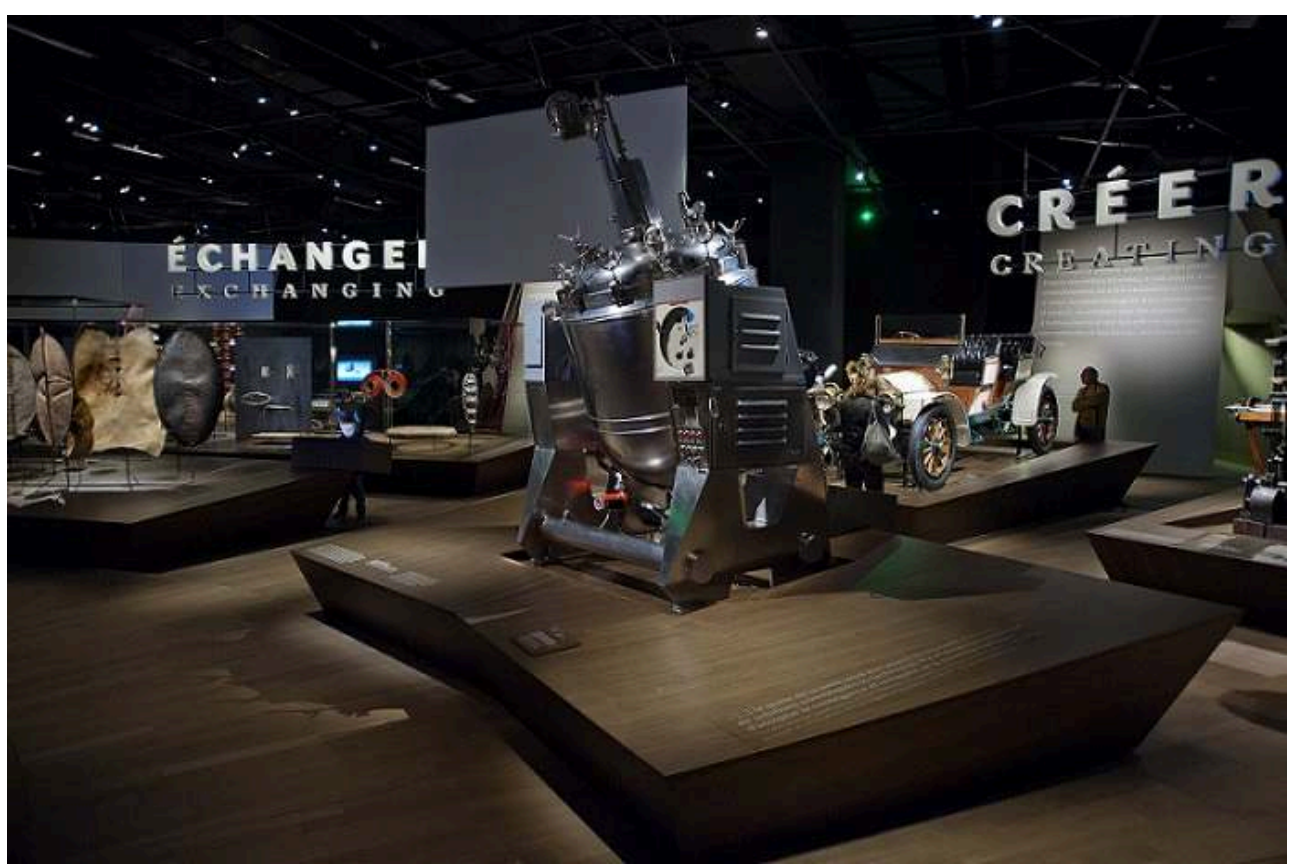

Les visiteurs sont invités à déambuler librement au sein des trois volets de ce chapitre intitulés

organiser, échanger, créer. Les collections exposées, très diverses, évoquent des questions propres à toutes les sociétés : leur structuration, le pouvoir, le territoire, l'innovation, les échanges...

Musée des Confluences, Lyon

L'exposition peut déstabiliser certains publics par le fait qu'aucun parcours n'est proposé. Certains construisent peu à peu un discours cohérent à partir des informations données dans les textes; mais d'autres préfèrent déambuler au hasard de la découverte d'objets curieux, spectaculaires ou particulièrement beaux. Les personnes mettant les pieds pour la première fois dans un musée -et la proportion au musée des Confluences est plus importante que dans d'autres- ne sont pas du tout gênées par la disposition de la salle; beaucoup se trouvent en terrain familier en découvrant grillepain, cocottes et robots ménagers. Dès lors, ils vont pouvoir partir à l'aventure, en se laissant guider par leurs seules envies, sans être contraints de suivre un parcours imposé. À ceux qui ne comprenaient pas le sens de cet amas apparemment désordonné de collection, j'ai régulièrement répondu par le recours à l'analogie, au modèle. En soi, cette partie de l'exposition permanente est un abrégé de notre monde, on y déambule comme dans une grande ville, découvrant tour à tour des véhicules de tous types, des monuments historiques, des constructions anciennes et actuelles, des gens de toutes origines, des magasins de mode et d'alimentation. L'approche est tout autre que pour la plupart des autres expositions, où un parcours est proposé avec un début et une fin, un cheminement où, comme dans un roman, l'histoire se construit page après page selon la volonté de l'auteur.

Ce caractère subjectif de la présentation, les choix proposés, imposés ou suggérés aux visiteurs ne sont pas le propre des musées de sociétés ou de sciences. Dans les musées d'art, la disposition des œuvres et le cheminement dans le musée sont aussi le résultat des choix scientifiques ou esthétiques des conservateurs. 


\section{L'authenticité, une exigence et un désir}

14 Je resterai un instant sur un autre exemple tiré du musée des Confluences pour introduire cette question centrale de l'authenticité. Lors de la présentation, en avantpremière, d'une partie du futur musée dans la grande salle de la Bourse du commerce de Lyon en 2010, nous avons choisi le thème Observer et exposé principalement des instruments scientifiques. Le but était de sensibiliser les publics, et en particulier les jeunes, à l'expérimentation et au rôle important des instruments dans les découvertes scientifiques.

Fig.4. Maquette à l'échelle 1 du rover martien ExoMars, présentée dans l'exposition Le musée des Confluences dévoile ses réserves, musée gallo-romain de Fourvière, Lyon, 2010-2011

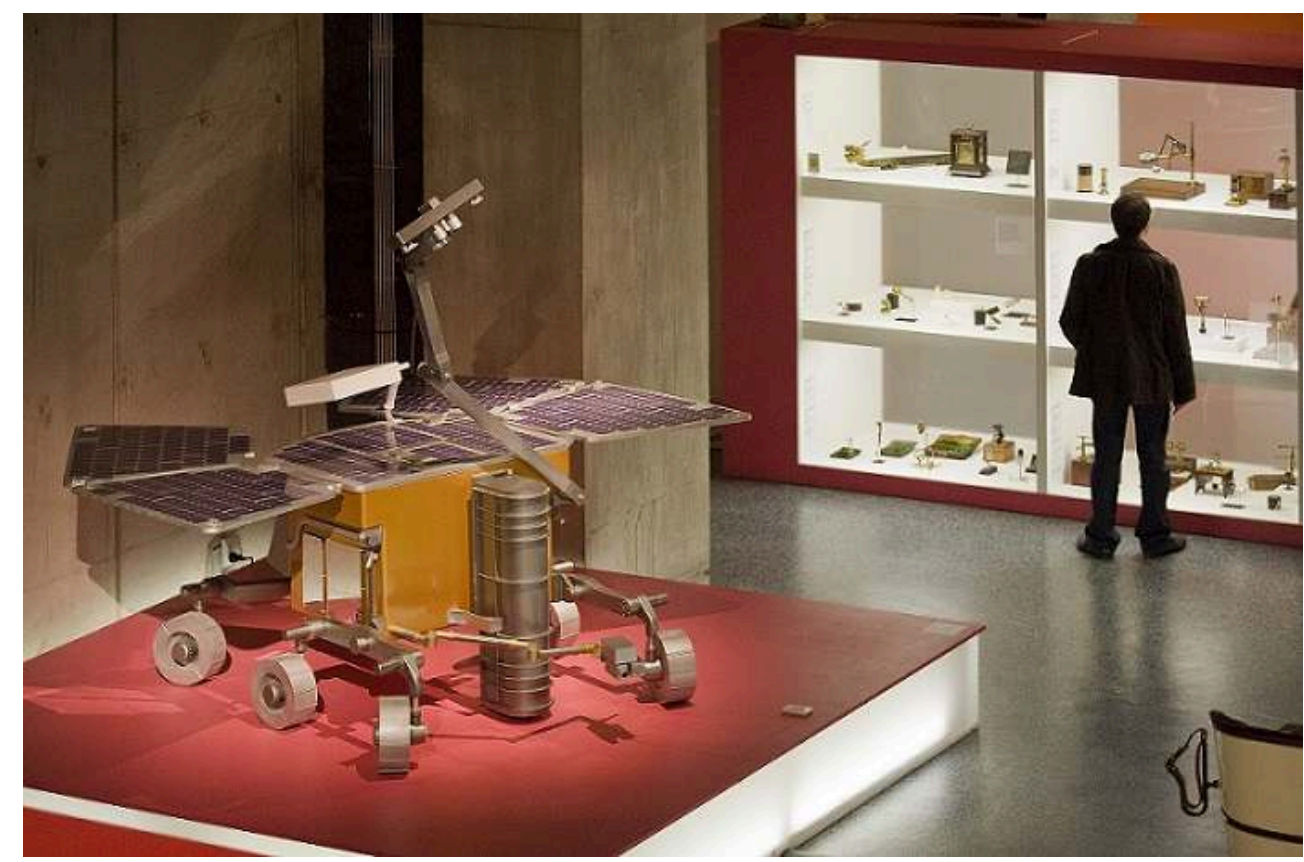

Une réplique à l'échelle 1 du rover ExoMars, fabriquée en 2008 par Hupkens Industrial Models, devait être présentée dans la salle Origines, sur le plateau consacré à la mesure du ciel et à l'exploration spatiale. Au bout du compte, elle n'a pas pu être exposée, notamment parce que la mission a été reportée. Une maquette de la sonde Huygens, acquise en même temps, est quant à elle suspendue au plafond au-dessus de la salle 3D. Le rover a été présenté pour la première fois par le musée des Confluences lors de l'exposition hors les murs Observer, à la Bourse du commerce de Lyon en février 2010.

Musée des Confluences, Lyon

Le parcours dans cette exposition faisait cheminer les visiteurs entre microscopes, télescopes, éléments de l'accélérateur de particules... et se terminait par la présentation des maquettes du rover martien ExoMars et de la sonde Huygens. Cette exposition avait notamment pour objectif d'étudier les pratiques, aspirations et attitudes des visiteurs, dont un certain nombre étaient invités à répondre à des entretiens à l'issue de leur visite. Parmi les conclusions de l'étude, j'ai été particulièrement frappé par celles relatives à l'authenticité. Découvrant en fin de visite deux objets qui étaient des reproductions et non des objets "authentiques ", le doute s'est immiscé chez certains quant à l'authenticité des autres instruments présentés dans la salle. En effet, c'est justement la présentation de pièces historiques authentiques qui est censée faire la différence entre les musées et les parcs d'attractions ou expositions commerciales, 
même si tout le monde comprend qu'on ne peut exposer des engins spatiaux originaux. Ces conclusions ont conforté l'équipe dans la nécessité de n'exposer qu'exceptionnellement des répliques, modèles ou copies.

Cela étant, la présentation de maquettes est perçue comme tout à fait légitime par le public dès lors que celles-ci ont acquis le statut d'objets historiques, ou s'agissant de la reconstitution d'objets qui ne sont plus accessibles autrement.

Pour illustrer le cas d'objets ayant pris une dimension historique a posteriori, je citerai l'exemple de la «maquette animée des usines du Creusot » exposée à l'écomusée de la communauté Le Creusot Montceau depuis 1983 (L'usine miniature 1984). Cette usine miniature, réalisée par un mécanicien du Jura au tournant du XXe siècle, montre une cinquantaine d'ouvriers forgeant au marteau-pilon, au marteau, laminant, sciant, etc.; cette attraction foraine tout à fait remarquable a circulé pendant plus de vingt années à travers la France et l'écomusée a pu l'acquérir auprès du dernier forain l'ayant exploitée, comme témoignage ethnographique de l'image de l'industrie métallurgique creusotine au XXe siècle.

Fig.5. Maquette animée des usines du Creusot, écomusée de la Communauté Le Creusot-Montceau

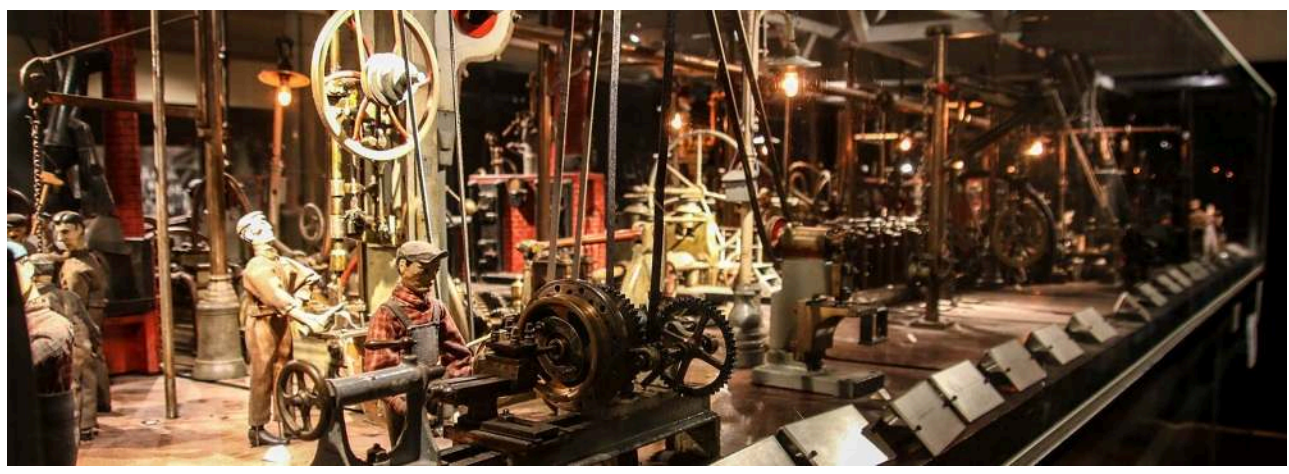

Cette maquette animée, aujourd'hui exposée à l'Ecomusée, a été réalisée par Joseph Beuchot, mécanicien aux forges de Fraisans (Jura) à la fin du XIXe siècle. On y voit notamment une grande forge avec le marteau-pilon et les laminoirs, une forge à main, des ateliers de construction et un atelier de menuiserie.

Écomusée de la Communauté Le Creusot-Montceau

Pour illustrer le témoignage d'éléments disparus, je pense bien sûr au musée des Monuments français ${ }^{7}$, dans lequel sont exposées des répliques grandeur nature des plus magnifiques tympans, fresques, éléments architecturaux de nombre de bâtiments historiques. Non seulement ces reproductions fidèles permettent à ses visiteurs, français ou étrangers, d'embrasser en une seule visite un nombre considérable de chefs-d'œuvre, mais certaines pièces sont aujourd'hui des témoignages irremplaçables de sculptures que les outrages du temps ont fortement dégradées ou détruites.

Cette question de l'authenticité se pose également pour des parties d'objets réalisées en complément de fragments existants, telles les poteries antiques, les fresques ou les pièces archéologiques. Rajouter un manche en bois ou en plexiglas à un marteau de bronze est le meilleur moyen de restituer la fonction de l'outil ; de même, on a recours aujourd'hui à des représentations virtuelles animées réalisées dans un but didactique. En l'occurrence, la doctrine en la matière, pour les musées de France, consiste à laisser les restaurations bien visibles afin que le visiteur ne soit pas trompé sur ce qui est authentique et ce qui a été ajouté. On sait toutefois combien ce principe connaît ou a 
connu d'écarts par le passé. Nombre de restaurations de tableaux ne sont aujourd'hui décelables qu'au moyen d'études approfondies non destructrices, les véhicules entrant dans les musées ont souvent subi de profondes transformations et la présentation de répliques de squelettes de dinosaure, par exemple, côtoie parfois des pièces authentiques sans que le visiteur en ait conscience, quand bien même la mention en soit faite sur les étiquettes des objets exposés.

Enfin, les maquettes, modèles ou dioramas jadis présentés comme des objets pédagogiques ont, avec le temps, acquis le statut de véritables objets historiques, comme les maquettes de Madame de Genlis au musée des Arts et Métiers, les dioramas géants du musée d'histoire naturelle de Berlin ${ }^{8}$, ou même les vitrines -les "unités écologiques »- crées par Georges Henri Rivière pour le musée national des arts et traditions populaires, aujourd'hui démontées, mais que nous avons eu le plaisir de retrouver successivement ces dernières années dans les expositions Dioramas à Paris en $2017^{9}$, Cabinets de curiosités à Landerneau ${ }^{10}$ et Georges Henri Rivière à Marseille en $2019^{11}$.

Fig.6. Vitrine « Du berceau à la tombe » reconstituée dans l'exposition Dioramas, Paris, Palais de Tokyo, 2017

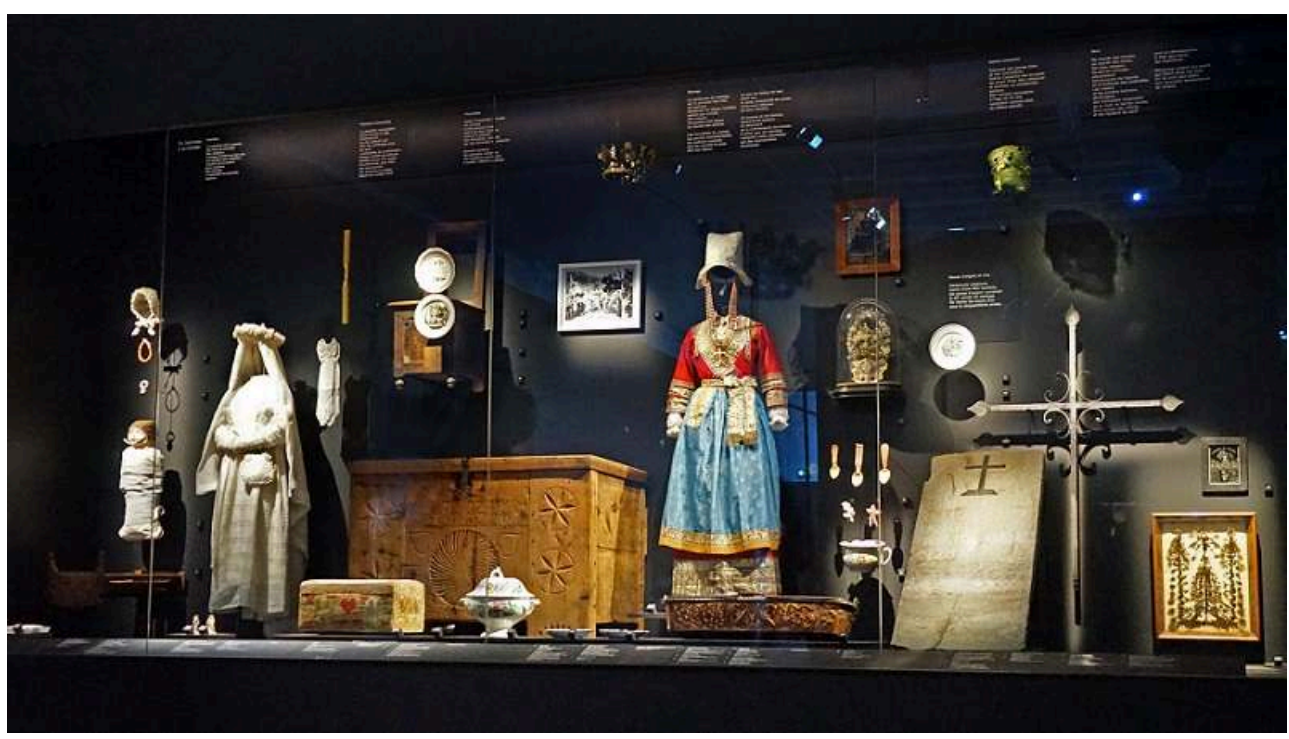

Cette vitrine emblématique de la muséographie de Georges Henri Rivière, initialement présentée dans la galerie culturelle du Musée national des arts et traditions populaires dès son ouverture en 1972, a été reconstituée dans l'exposition Dioramas (commissaires : Claire Garnier, Laurent Le Bon, Florence Ostende).

Jean-Pierre Dalbera

\section{Le choix de l'objet}

L'authenticité est donc un critère déterminant pour le choix des objets qui sont exposés, mais ce n'est pas le seul, loin de là. Le temps où l'on présentait dans des salles l'intégralité d'une collection est révolu. Nombre de musées n'exposent guère plus d'un dixième de leur fonds. Dès lors, la sélection réalisée pour illustrer un discours ou montrer les richesses d'une collection est le résultat d'une délicate alchimie, guidée à la fois par la volonté de l'institution et celle, plus personnelle, du concepteur. 

scientifique. J'entends par spectaculaire un objet qui, par la taille, la couleur, les matériaux, va arrêter la pérégrination du visiteur, le contraindre en quelque sorte à se questionner, se poser un instant et prendre le temps de regarder les objets environnants, que sans cela il n'aurait pas remarqués. La notion de rythme dans une exposition est capitale. Si tout est sur le même plan, de la même taille, d'une catégorie semblable pour le visiteur, celui-ci risque de passer son chemin sans saisir l'intérêt de certaines pièces plus discrètes. D'où l'utilité de présenter un squelette de mosasaure de quatorze mètres de long, un supercalculateur Cray avec son refroidissement liquide, une machine à vapeur peinte de couleurs vives, ou un casque de général japonais du XVI ${ }^{\mathrm{e}}$ siècle ressemblant à celui de Dark Vador... authentiques et en bon état, on privilégiera le plus beau des deux, car le visiteur vient au musée pour le plaisir autant que pour apprendre. Mais il s'agit bien sûr d'un critère subjectif, car il dépend beaucoup de la culture du visiteur. Telle personne s'extasiera devant une sculpture inuit et une autre face à une Formule 1 Renault !

27 Je terminerai enfin ma liste par un critère encore plus subjectif, celui de l'étrangeté. Il s'apparente au caractère spectaculaire, mais il dépend beaucoup de la culture de chacun, et va de pair avec un caractère de familiarité. J'ai souvent écouté les réactions de visiteurs devant la vitrine des micro-ordinateurs domestiques dans le domaine Communication du Musée des arts et métiers. Combien de fois ai-je entendu quelqu'un 
expliquer à sa famille ou à ses amis que tel appareil avait engendré une véritable révolution dans l'histoire de la micro-informatique, uniquement par le fait qu'il avait représenté, pour cette personne, une acquisition qui avait changé le cours de son existence ? Et c'est très bien ainsi. Le dialogue entre l'objet et le visiteur commence souvent par un regard familier, voire bienveillant.

Évidemment, cette liste de critères n'est pas exhaustive, et surtout il serait illusoire d'en mesurer la proportion respective dans le cadre d'une sélection d'autant que ces critères sont constamment en conflit les uns avec les autres. Ce calculateur analogique est pertinent, mais ne parle à personne, ces échantillons de soie sont magnifiques et représentatifs, mais ne supporteront pas la lumière, etc.

\section{Le musée, espace de liberté}

Nous venons de voir combien le choix des objets exposés est subjectif. Et il est lui-même soumis au propos des concepteurs. Je préfère ce terme à celui de discours, parfois utilisé, car il ne s'agit finalement que de proposer, et non d'imposer. Quoi qu'on fasse, qu'on montre et qu'on écrive, le visiteur est au bout du compte l'auteur de sa propre visite, de sa propre histoire. Il en est de même pour l'espace de l'exposition et la manière dont sont disposés œuvres, objets, manips, etc. Il est impératif de prendre en compte la liberté du visiteur de ne pas lire, de prendre l'exposition à rebours, ou de s'asseoir dix minutes devant tel objet qu'on a pu juger tout à fait secondaire. La proposition ne doit pas être neutre pour autant et, même si cela est inconscient, la disposition muséographique révèle la personnalité des concepteurs ou promoteurs. La présence ou l'absence de vitrine, la possibilité de tourner autour de l'objet, l'intimité créée par un éclairage discret, tous ces éléments infléchissent le regard que le visiteur porte sur ce qu'on lui présente.

Je clôturerai ses réflexions sur " le sens de la visite ${ }^{12}$ " par l'évocation de la notion de temps. Plusieurs temps différents se superposent dans le cadre de la visite. Le temps de l'histoire d'abord : il s'agit du temps long, celui qui scande l'organisation de nombre de musées ou de parties d'entre eux. Le déroulement chronologique d'une exposition permet de rendre compte de l'évolution générale des sociétés, de la science, des connaissances humaines ou des arts. Au Musée des arts et métiers, chacun des sept grands domaines qui structurent l'exposition permanente est rythmé par le même découpage en quatre périodes dont les limites sont les dates de 1750, 1850 et 1950. Ces dates charnières ont fait l'objet d'âpres discussions au sein du comité scientifique avant d'être inscrites dans la topographie du musée. Le but de ce découpage est de permettre aux visiteurs de se situer dans un contexte social, économique et technique donné, tout en offrant la possibilité de se repérer d'un domaine à l'autre. Mais la disposition des lieux autorise tout un chacun à parcourir les espaces d'exposition dans le sens qui lui plaît, même si la proposition principale va dans le sens naturel de l'histoire.

31 Le cas de la salle Origines du musée des Confluences a fait l'objet d'une proposition plus audacieuse, que nous avions déjà envisagée pour le Musée des arts et métiers mais qui ne pouvait être mise en œuvre dans ce contexte spécifique. Dans Origines, le parcours, en l'occurrence imposé pour des raisons de gestion des flux de visiteurs, a été délibérément antichronologique. L'exposition commence par la découverte de trois espèces humaines qui ont cohabité longtemps -ici représentées par des reconstitutions des trois femmes Sapiens, Néandertal et Flores- puis le parcours remonte le temps 
jusqu'aux origines de la vie et se termine par l'évocation du big bang. Ce choix présente l'atout majeur d'éviter un écueil présent dans les présentations anciennes de nombreux muséums : celui de suggérer l'idée fausse que l'avènement de l'espèce humaine serait déterminé par une logique inéluctable tendant à faire de notre espèce, au terme d'un long cheminement, la plus noble création de l'univers, voire de Dieu. Or, on sait à présent par quelle extraordinaire succession de hasards, de catastrophes, de phénomènes naturels cette espèce est parvenue à ce qu'elle est aujourd'hui. Le recours à un parcours antichronologique permet de situer notre espèce non pas au sommet d'une pyramide de l'intelligence ou de la complexité, mais à l'extrémité d'un tout petit rameau d'un gigantesque "buisson du vivant ", représenté graphiquement à chaque étape de ce grand récit de l'évolution.

32 Au Musée des arts et métiers, le temps long de l'histoire se double d'un autre rythme de durée variable: le temps de l'innovation. De la volonté même de ses fondateurs, le Conservatoire des arts et métiers est le temple de l'innovation technique. D'où la grande proportion de maquettes pédagogiques, de prototypes, de machines qui, sans avoir de descendance directe, n'en ont pas moins eu un avenir par le biais des techniciens, ingénieurs et artisans qui sont venus y glaner matière à inventer et à innover. S'agissant donc de s'inscrire dans ses principes fondateurs, il a été décidé de disposer, en plusieurs endroits, des séries cohérentes d'objets de la même famille dans lesquelles les visiteurs ont la possibilité de décrypter le cheminement de la pensée technique des créateurs successifs. À la manière de Queneau, remontons le temps pour reconstituer cette filiation: "Quelle est son origine ? En partant de l'objet // Retrouvons ses aïeux ! Qu'à l'envers se déroule // son histoire exemplaire ${ }^{13}$. »

La durée relative de ces micro-histoires peut être très variable : quelques siècles pour les ponts ou les microscopes, quelques dizaines d'années pour les piles électriques ou les vélos. Permettre au visiteur de se mettre « dans la peau » du créateur est à mon avis la meilleure manière d'établir ce dialogue entre les objets et les humains. 
Fig.7. Vitrine des microscopes. Musée des arts et métiers, Paris

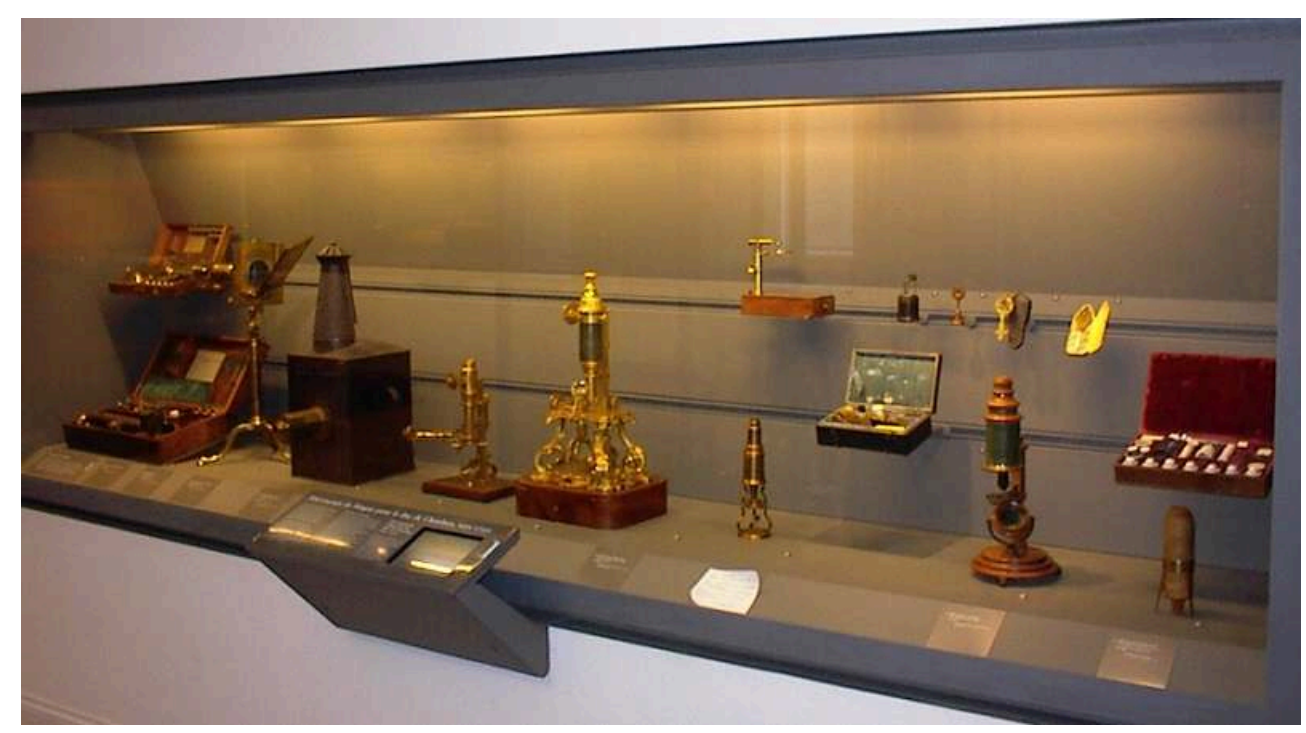

Dans cette vitrine, présente dans le domaine des Instruments scientifiques du Musée des arts et métiers, sont exposés plusieurs types de microscopes : simples, composés, de projection. Une première lecture montre la variété des formes et techniques des objets ; une observation plus approfondie, menée avec l'aide d'un démonstrateur, permet de décrypter les lignées techniques et l'évolution des différents types au cours du temps.

Bruno Jacomy, Musée des arts et métiers, Paris

Enfin, il existe un troisième type de temps au musée : celui de la visite. Celui-ci n'était aucunement contraint. Dans les différents lieux où j'ai exercé, je me suis souvent trouvé à répondre à une question simple: combien faut-il de temps pour visiter le musée ? Ma réponse était plutôt du type : « si vous voulez tout lire et tout voir, comptez une semaine, mais si vous n'avez qu'une heure ou deux, n'hésitez pas à faire un tour pour sentir l'atmosphère du lieu, ou à choisir l'une des salles proposées.» De toute manière, la durée de la visite est davantage guidée par le temps de la fatigue physique que par le volume des choses à voir.

\section{Conclusion}

Quoi qu'il en soit, chacun a sa manière propre de visiter un musée ou une exposition. Le principal n'est pas la masse de connaissances que les visiteurs vont pouvoir acquérir lors de leur passage au musée, mais l'envie d'en savoir plus et le plaisir de la découverte. Et ceci passe par un dialogue entre objets et visiteurs, par le truchement ou non d'un médiateur, et par cette intimité qui va rendre le lieu familier.

\section{BIBLIOGRAPHIE}

«L'usine miniature », Milieux, n 15-16, octobre 1983-janvier 1984, p.15-49 
CARRIÈRE Jean-Claude et SÉMÉNIAKO Michel, Le sens de la visite, Paris, Éd. Carré, Musée des arts et métiers, 1997

HAINARD Jacques, « La muséologie du point de vue », dans CÔTÉ Michel (dir.), La fabrique du musée de sciences et sociétés : actes des journées d'étude "L'exposition de sciences et sociétés, particularités, tendances et enjeux », Paris, La Documentation française, 2011, p.11-13

DAVALLON Jean, « Penser l'exposition comme rituel de représentation », dans DAVALLON Jean (dir.), Claquemurer, pour ainsi dire, tout l'univers : la mise en exposition, Paris, Centre Georges Pompidou, Centre de création industrielle, 1986, p.269-279

MELOT Michel, « Bâtons rompus sur l'exposition », dans CARRIER Christian, L'objet expose le lieu : présentation, représentation, exposition, Paris, Expo Média, 1986, p.9-17

Musée des Confluences: Guide, Lyon, musée des Confluences, éd. Courtes et Longues, 2014

WAGENSBERG Jorge, « What does Creating a Museum of Science through Conversation Mean? », dans WAGENSBERG Jorge, TERRADAS Robert et ARNAL Marc (dir.), Cosmocaixa: The Total Museum through Conversation between Architects and Museologists, Barcelona, Sacyr, 2006, p.72-97

\section{NOTES}

1. « Savoir signer et avoir du style », suggère Jacques Hainard (Hainard 2011).

2. Jorge Wagensberg, fondateur du Cosmocaixa de Barcelone, un des plus remarquables centres de sciences actuels, fonde son concept de "muséologie totale " sur l'idée de "conversation » (Wagensberg 2006). Pour lui, la visite au musée doit changer votre vie ("the main function of a museum is that the visit changes your life"), p. 27.

3. Michel Melot cite cette formule de Pierre Georgel, commissaire de l'exposition La Gloire de Victor Hugo, Grand Palais, 1985-1986 (Melot 1986).

4. Dans la chambre des merveilles, musée des Confluences, Lyon, 2014-2016 (commissaires : Joël Clary et Bruno Jacomy; scénographie: Ève-Marine Basuyaux, Florence Reibell et Tiphaine Massari) .

5. J'emprunte cette belle formule au titre de l'exposition présentée à l'Institut national d'histoire de l'art en 2012 : 1740, un abrégé du monde, savoirs et collections autour de Dezallier d'Argenville (commissaire : Anne Lafont).

6. Équipe projet: Christian Sermet, Carole Millon, David Besson. Comité scientifique : Roland Bacon, astrophysicien, Élisabeth de Fontenay, philosophe, Pierre Gibert, théologien, Pascal Picq, paléoanthropologue, Pierre Thomas, géologue.

7. Né musée de Sculpture comparée en 1882, il devient musée des Monuments français en 1937 et s'installe dans une aile du Palais de Chaillot à Paris ; il est intégré à la Cité de l'architecture et du patrimoine depuis 2004 .

8. Museum für Naturkunde, Berlin.

9. Dioramas, exposition au Palais de Tokyo, Paris, 2017 (commissaires : Claire Garnier, Laurent Le Bon et Florence Ostende).

10. Cabinets de curiosités, exposition au Fonds Hélène \& Édouard Leclerc pour la Culture, Landerneau, 2019 (commissaire : Laurent Le Bon).

11. Georges Henri Rivière. Voir c'est comprendre, exposition au MuCEM, Marseille, 2018-2019 (commissaire : Germain Viatte).

12. J'emprunte cette judicieuse formule au titre de l'ouvrage (Carrière, Séméniako 1997).

13. Extrait du poème écrit par Raymond Queneau pour le court-métrage Le Chant du styrène, réalisé par Alain Resnais en 1958. 


\section{RÉSUMÉS}

À l'image des compendiums scientifiques des anciennes écoles élémentaires, le musée présente une collection de choses rassemblées au nom d'un objectif commun, unis par une trame plus ou moins explicite. Ce monde en réduction est mis en scène, telle une représentation théâtrale, dans les limites physiques de l'espace du musée et du temps de la visite. Mais cette question de la représentation renvoie immanquablement à celle de l'authenticité, que posent notamment maquettes, modèles, dioramas, reconstitutions en tout genre, ainsi qu'à celle du temps qui passe. Ce dernier est multiple dans le musée ou l'exposition; le temps fugace de la visite, le temps long de l'histoire ou celui, circonscrit, de l'innovation technique, s'entrecroisent et se répondent dans cet espace clos dédié à la découverte et à l'imagination. Si les musées -et tout particulièrement les musées de sciences, de techniques ou de sociétés- se doivent d'être objectifs, ils n'en sont pas neutres pour autant. Les conservateurs, commissaires d'expositions ou muséologues proposent un ordre, une trame, une scénographie, mais le visiteur reste maître du jeu car lui seul décide du temps de sa visite et du récit qu'il compose à partir des objets qu'il rencontre. Au bout du compte, le musée, espace multiple par ses collections et collectif par ses visiteurs, ne se résume-t-il pas à un échange unique et intime entre un objet et un individu, entre une œuvre et un regard?

Like the science books of the old elementary school, the museum presents a collection of things gathered in the name of a common objective, united by a more or less explicit framework. This reduced world is staged, like a theatrical representation, within the physical limits of the museum space and the time of the visit. But this question of representation inevitably refers to that of authenticity, posed in particular by the models, dioramas, reconstitutions of all kinds, as well as to that of the passing of time. The latter is multiple in the museum or the exhibition; the fleeting time of the visit, the long time of history or the circumscribed time of technical innovation, cross and respond to each other in this closed space dedicated to discovery and imagination. If museums - and more particularly science, technology or society museums - must be objective, they are not neutral. The curators or museologists propose an order, a framework, a scenography, but the visitor remains the master of the game because he decides alone the moment of his visit and the story he composes from the objects he meets. In the end, isn't the museum, a multiple space for its collections and a collective space for its visitors, similar to a unique and intimate exchange between an object and an individual, between a work of art and a way of looking at it?

\section{INDEX}

Mots-clés : histoire des techniques, musée, muséologie, objet technique, scénographie

Keywords : history of technology, museum, museology, technical object, scenography

\section{AUTEUR}

\section{BRUNO JACOMY}

Bruno Jacomy est conservateur en chef honoraire du patrimoine ; il a assuré la direction scientifique du Musée des Confluences, à Lyon, de 2005 à 2016. Ingénieur arts et métiers (ENSAM) de formation, il a été conservateur à l'Écomusée du Creusot-Montceau (1976-1983) et a assuré, de 1989 à 2005, la responsabilité scientifique de la rénovation du Musée des arts et métiers à Paris. Il 
a notamment publié Une histoire des techniques (Seuil, 1990 et 2015) et L'Âge du Plip : chroniques de l'innovation technique (Seuil, 2002). Il a soutenu en 1983 une thèse de psychologie sociale et a assuré, depuis 1990, des cours et conférences à l'UTC (Compiègne) et dans plusieurs masters de muséologie et d'histoire des techniques (CNAM, Muséums, Université Jean Monnet de SaintÉtienne...). 